\title{
Proposal of super fast charge and easy battery exchange mechanism for battery-powered equipment
}

\author{
Shun Ito ${ }^{\text {a, }}$, Ryunosuke Tadoh ${ }^{a}$, Seiichi Serikawa ${ }^{a}$ \\ ${ }^{a}$ Kyusyu Institute of Technology, Kitakyushu, 804-8550, Japan \\ *Corresponding Author: sh-lo-y@i.softbank.jp
}

\begin{abstract}
Recently, in Japan, an idea of IoT (Internet of Things) is spreading. Then, an opportunity to put a sensor at the various places increases. And if we use an outlet for domestic for the power of it, the place where a sensor will be put is limited. Therefore, IoT equipment of the battery type is important to the spread expansion of IoT in the future because it doesn't limit the place where a sensor is put. However, IoT equipment of the battery type has also disadvantages. Specifically, it is "when we manage many IoT equipment, it takes much time for an exchange of batteries" and "charge time is long". Therefore, the purpose of this research is the improvement of the two disadvantages of its battery type.

First, an improvement to "when we manage many IoT equipment, it takes much time for an exchange of batteries" is to easily exchange by using a contact type and a magnet for a bonding plane of a pole of a battery and a pole of a charge.

Second, an improvement to "charge time is long" is using a capacitor.
\end{abstract}

Keywords: capacitor, easily exchange

\section{Introduction}

In recent years, the idea of IoT (Internet of things) is spreading in Japan. As the spread of IoT spreads, opportunities to put sensors in various places increase. However, when using a household power outlet for the power supply, it takes time to draw the code. Furthermore, depending on the installation location of the equipment, construction is also required. Then it costs money. Therefore,

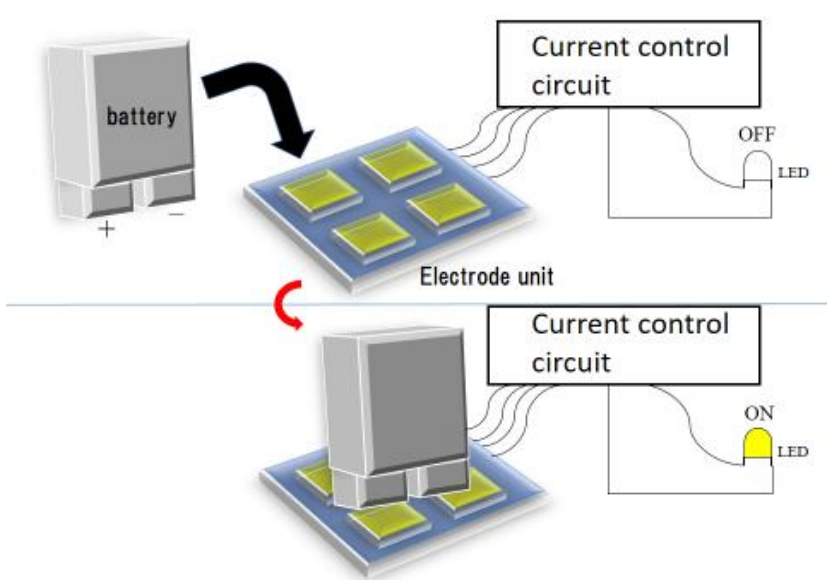

Fig. 1 Prediction of completion of the mechanism of this research.

battery type IoT equipment is expected to be used in many places in the future.

However, there are two disadvantages in battery type. First, in recent years research on energy saving of batteries has been advanced, but still it is necessary to replace them, and it takes time in that case. Second, it takes time to charge the battery. Therefore, this research aims to solve the disadvantage of battery type.

First, the first disadvantage of " it takes much time for an exchange of batteries " solution is to bring the electrode on the battery side into contact with an arbitrary position of the electrode on the power supply circuit side in order to facilitate battery replacement In order to supply power to the device by using only the power supply circuit. A completed projection is shown in Figure 1.

Also, regarding the second disadvantage of "it takes time to charge the battery ", we aims to shorten the charging time by enabling large current charging by using a capacitor as a battery. 


\section{Principle}

\subsection{Power supply circuit}

First, Figure 2 shows the principle diagram of the power supply circuit that supplies power from the battery to the device. In this study, we constructed a quadrangular electrode plate in contact with the electrode of the battery with a pair of four electrode plates, and further connected current control circuits with them to enable the current to flow only in a certain direction to the device side. In addition, even when the electrode of the battery straddles and contacts a plurality of electrode plate, the current flows only in a certain direction, so there is no restriction on the place where the battery is placed.

\subsection{Electric double layer capacitor}

A capacitor used as a battery for shortening the charging time will be described. Since this time it is used as a battery, an electric double layer capacitor with high energy density among capacitors was used. Although it is inferior to secondary batteries in terms of energy density, charging and discharging are carried out by movement of ions, so there is almost no degradation and the life is long.

\subsection{Constant current circuit}

Figure 3 shows the constant current circuit used to verify the charge time this time. Using this circuit, constant current charging was performed at about 2.5 ampere.

\section{System configuration}

\subsection{Power supply circuit}

The system configuration of the power supply circuit will be described. First, Figure 4 shows the circuit diagram of the power supply circuit including the details of the current control circuit shown in Figure 2. By constructing this circuit, it is possible to flow a current in a certain direction to the device side (output side) regardless of the contact location of the electrode plate of the electrode on the input side and the number of contacts with the electrode plate. This mechanism is realized by using a bridge diode having a function of rectifying an alternating current to a current control circuit to make it a direct current. In this example, current rectification is performed by connecting two bridge diodes in parallel to four electrode plates.

\subsection{Electrode unit}

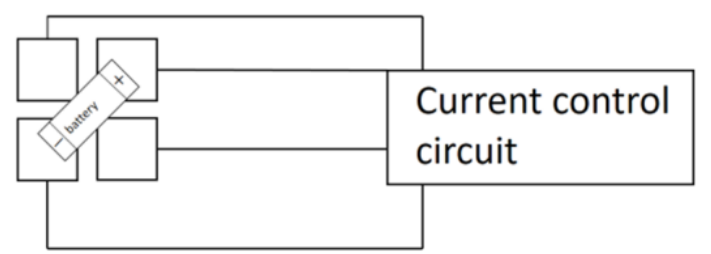

Fig. 2 Principle of the power supply circuit of this research.

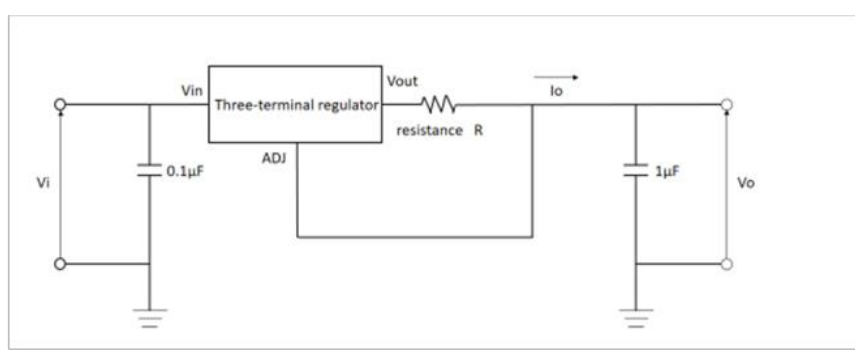

Fig. 3 Constant current circuit used in this study.

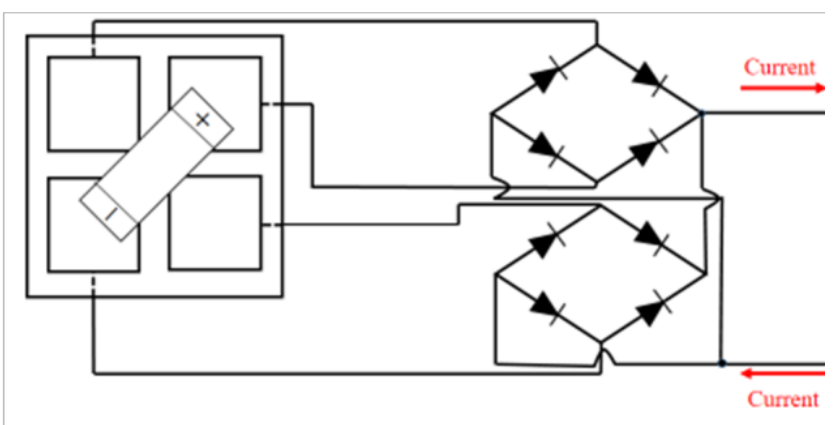

Fig.4 Circuit diagram of power supply circuit including details of the current control circuit shown in Figure 2.

In this research, a contact type electrode plate is used in the power supply circuit for supplying power to the device. However, when placing the battery there, there should be no short circuit or a situation where the electrode of the battery does not contact the electrode plate. Therefore, it is necessary to set conditions for preventing such troubles in the size of the electrode of the battery and the size of the electrode plate. Figures 5 and 6 show a pattern not in contact and a shortcircuited pattern. The variables of the battery and the electrode unit are shown in Figures 7 and 8. By using this variable, the conditional expression that does not cause a pattern not in contact and a short circuit is expressed as follows.

- Condition that no short circuit occurs

$$
\mathrm{L}>\sqrt{w^{2}+h^{2}}
$$



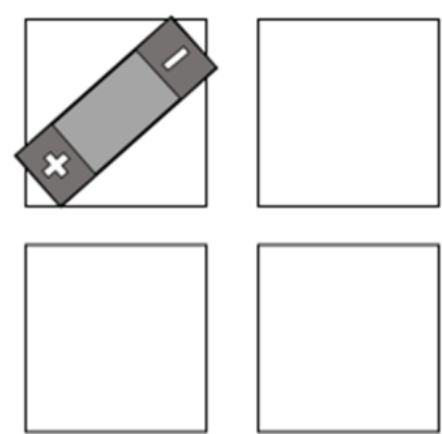

Fig. 5 Short circuit pattern drawing.

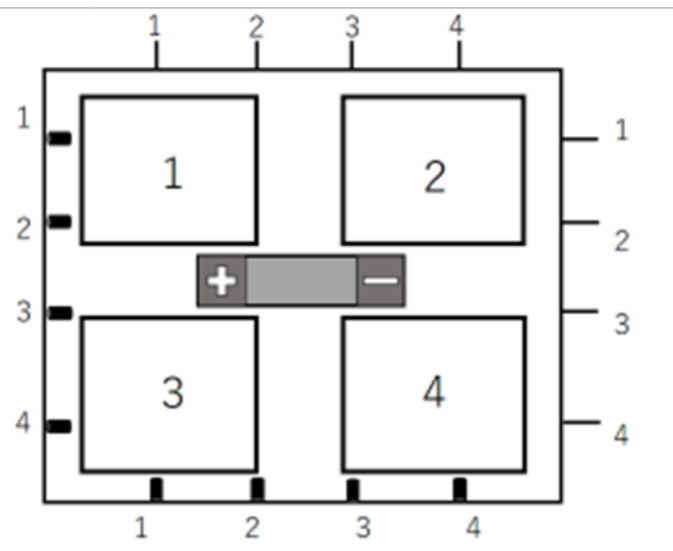

Fig. 6 Pattern in which the electrode of the battery does not contact the electrode plate.

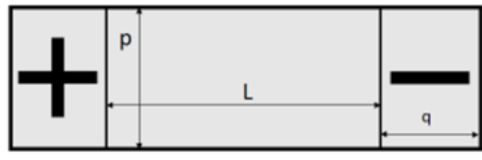

Fig. 7 Schematic diagram of the battery.

- Conditions which no contact pattern does not occur

$$
0<\sqrt{2} d<\min (p, q)
$$

Also, in Figure 8, male and female electric wires are connected to the unit of the electrode plate. This is attached so that it can be expanded in order to avoid that the electrode plate can be used only with a specific battery. Electrical wires of the same number are connected to electrode plates, and when expanded, electrode plates of the same numbers are connected in parallel. By doing so, it is possible to expand the electrode. Also, the pattern in which a short circuit occurs

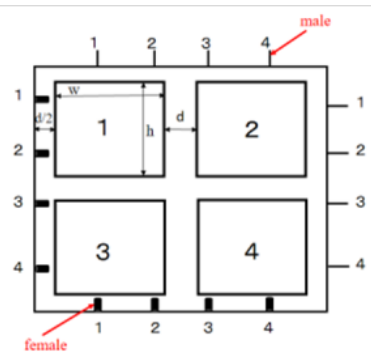

Fig. 8 Schematic diagram of electrode unit.

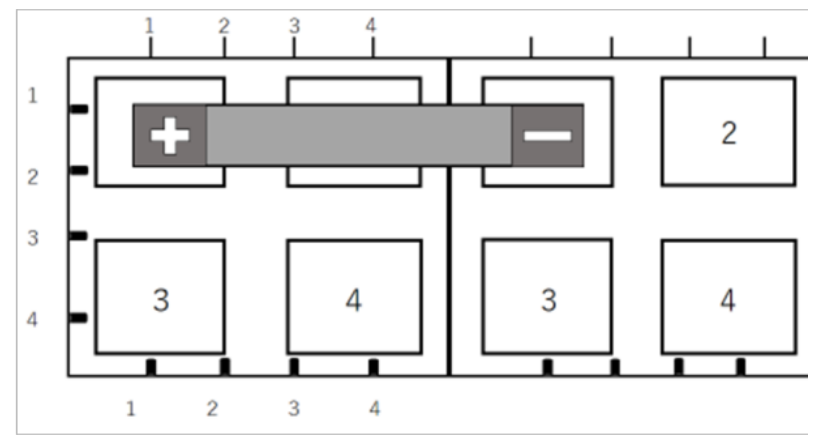

Fig. 9 Short circuit pattern when electrode unit is expanded.

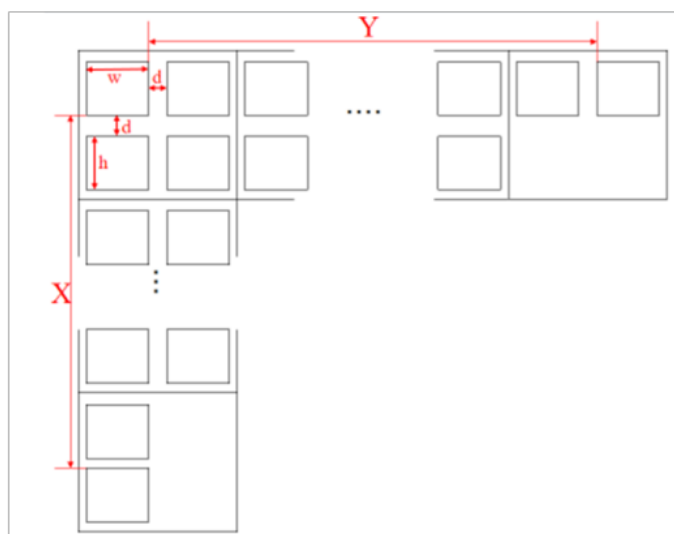

Fig. 10 Expansion of electrode toward $\mathrm{X}$ and $\mathrm{Y}$ direction.

when expanding is shown in Figure 9.

Here, $\mathrm{X}$ and $\mathrm{Y}$ when the electrode unit is expanded are determined as shown in Figure 10. In this case, if it is assumed that $\mathrm{n}$ electrode units are expanded in the $\mathrm{X}$ direction, then the length of $\mathrm{X}$ is

$$
X=(2 h+2 d) n-2 h-d
$$

.Likewise, the length in the $\mathrm{Y}$ is

$$
Y=(2 w+2 d) n-2 w-d
$$

.Therefore, as long as the lateral length $(L+2 q)$ of the battery is larger than the longer one of the lengths of $\mathrm{X}$ and $\mathrm{Y}$,

$$
\mathrm{L}+2 \mathrm{q}>\max (\mathrm{X}, \mathrm{Y})
$$




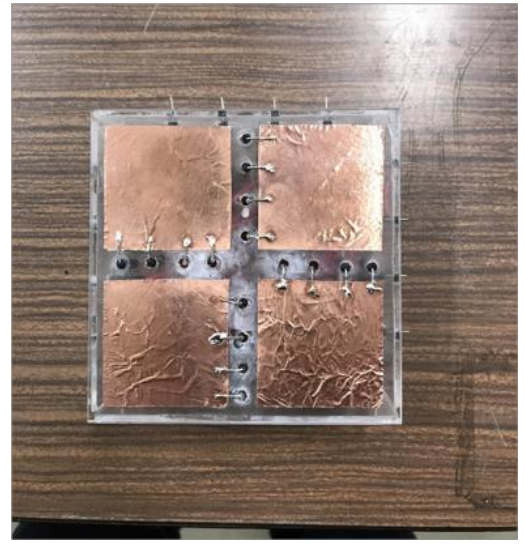

Fig. 11 The prepared electrode unit.

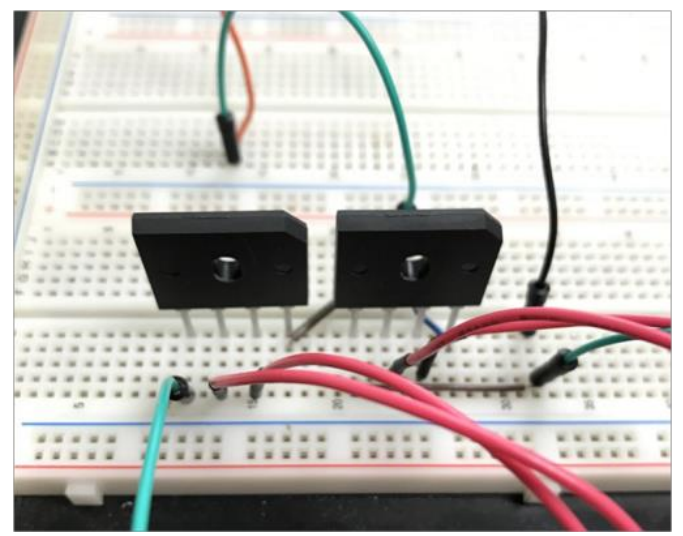

Fig. 12 Circuit of bridge diode section

\section{Circuit fabrication}

The electrode unit fabricated this time is shown in Figure 11 , and the circuit of the bridge diode part is shown in Figure 12.

Also, the electrode at the time of expansion is shown in Figure 13.

Figure 14 shows the battery made by the capacitor in this study. (a) is a picture of the battery viewed from the side, and (b) is a picture taken from the electrode side of the battery.

\section{Experiment}

As an experiment in the power supply circuit, first, experiments were conducted to check whether the four electrode plates (1 unit) function properly, that is, whether

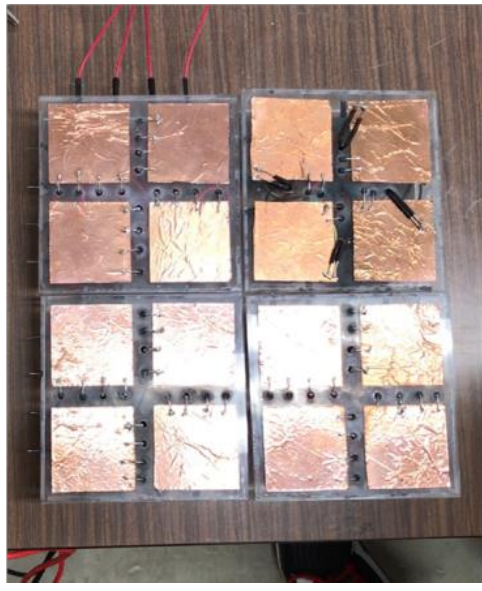

Fig. 13 Electrode plate of power supply circuit during expansion.

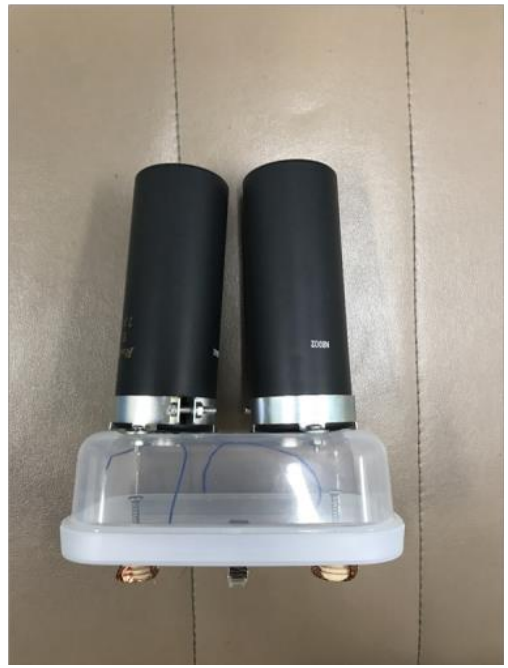

(a)

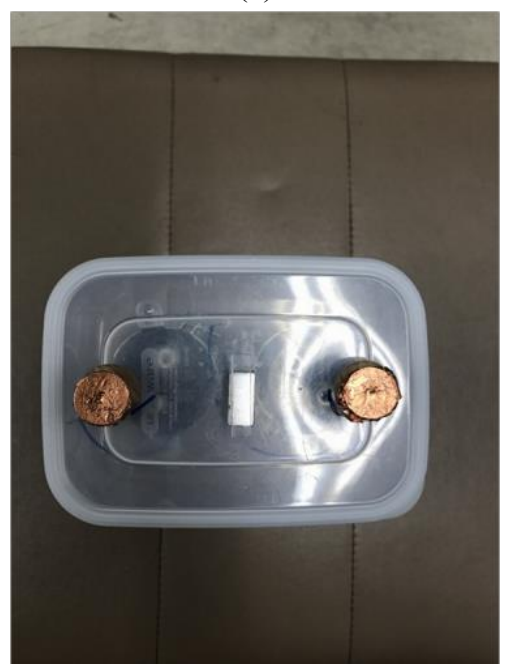

(b)

Fig. 14 Battery made by capacitor prepared in this study. (a) is a picture of the battery viewed from the side, and (b) is a picture taken from the electrode side of the battery. 
the current of the device is flowing no matter where the electrode of the battery is placed. As a content, voltage was actually applied to all patterns where the battery electrode and the four electrode plates were in contact, and it was confirmed whether current was flowing. There are 32 patterns in all. As a result of the experiment, it was confirmed that current flows in all patterns. Therefore, it was confirmed that the current supply circuit fabricated this time was a circuit capable of flowing current in a certain direction regardless of the contact location between the battery electrode and the electrode plate.

In the next experiment, even when the electrode plate was expanded, it was confirmed whether the current could flow in a certain direction regardless of where the battery was installed. As a confirmation method of the current flow, it was confirmed whether the current was flowing in a certain direction by turning on the LED. This time, expansion was carried out using four electrode plate units. We also confirmed the versatility of the power supply circuit by using the batteries equipped with the electric double layer capacitor which were prepared in this study and the commercially available rechargeable battery.

As a result of the experiment, in both batteries, except for the short circuit pattern mentioned in Chapter 3. It was possible to confirm the lighting of the LED irrespective of the touch location, and it was possible to confirm the versatility of the electrode unit.

Next, verification experiments on charging of batteries using electric double layer capacitors were carried out. Approximately 2.5 amperes of charge using the constant current circuit in Figure 3 took about 34 minutes to reach the same capacity as 950 [mAh]. On the other hand, since the charging time of the eneloop lite $(950 \mathrm{mAh})$ is about 6 hours, it was confirmed that by using a capacitor for the battery, it is possible to greatly shorten the charging time.

\section{Conclusion}

The research aimed to solve the disadvantage of the battery to expand the diffusion of battery type IoT in the future, "it takes much time for an exchange of batteries "," it takes time to charge the battery "

As a result, by using the power supply circuit fabricated this time, it is possible to send current to the device simply by placing the battery on an arbitrary position of the electrode plate regardless of the location of contact between the battery electrode and the electrode plate on the supply circuit It was possible to obtain the advantage that the replacement of the battery becomes easy.

In addition, by using an electric double layer capacitor with a high energy density in the capacitor for the battery, it is possible to eliminate the limitation of the charging current and to charge with a large current, and as a result of experiments, it is possible to obtain a large charging time as compared with a commercially available rechargeable battery reduction in the amount of waste can be realized.

For future work, we could not produce and experiment with magnet-based devices. Therefore, we do that verification.

\section{References}

(1) Mayonez, "Japan IoT utilization case 20 pieces", h ttps://mayonez.jp/topic/1492

(2) Ministry of Public Management, "From ubiquitous to IoT", http://www.soumu.go.jp/johotsusintokei/whit epaper/ja/h27/html/nc254110.html

(3) RS Electronics Center, "Electric Double Layer Cap acitor Edition", https://jp.rs-online.com/web/generalD isplay.html?id=centre/eem_mrtech1108

(4) Panasonic, “eneloop", http://panasonic.jp/battery/char ge/eneloop.html

(5) Okamura Takeo, Kinoshita Shigeru, "Characteristics and Good Use of Electric Double Layer Capacitor $<$ EDLC > ", Nikkan Kogyo Shimbun, pp.1 - 199 (2010)

(6) Okamura Takeo, "Electric Double Layer Capacitor and Storage System (2nd Edition)", Nikkan Kogyo Shimbun, pp.1-213 (2003) 